\title{
Ophthalmic Imaging Tools to Detect Glaucoma Progression
}

\author{
Tigran Kostanyan, MD¹ and Gadi Wollstein, MD² \\ 1. Research Fellow; 2. Associate Professor of Ophthalmology, UPMC Eye Center, Eye and Ear Institute, Ophthalmology and Visual Science Research Center, \\ Department of Ophthalmology, University of Pittsburgh School of Medicine, Pittsburgh, Philadelphia, US
}

\begin{abstract}
Ocular imaging devices provide objective and reliable structural measurements and estimate structural progression that has important clinical implications in ocular pathologies such as glaucoma. This review describes the working principles and main methods for detection of glaucoma progression used in the leading commercially available ocular imaging. This information allows clinicians to better identify eyes that are suspicious of progression and adjust the clinical management as needed.
\end{abstract}

\section{Keywords}

Spectral domain optical coherence tomography (SD-OCT), confocal scanning laser ophthalmoscopy (CSLO), scanning laser polarimetry (SLP), glaucoma progression

Disclosures: Tigran Kostanyan, MD, and Gadi Wollstein, MD, have no conflicts of interest to declare. No funding was received in the publication of this article. Open Access: This article is published under the Creative Commons Attribution Noncommercial License, which permits any noncommercial use, distribution, adaptation, and reproduction provided the original author(s) and source are given appropriate credit.

Received: August 30, 2014 Accepted: October 10, 2014 Citation: US Ophthalmic Review, 2015;8(1):37-42 DOl: 10.17925/usor.2015.8.1.37

Correspondence: Gadi Wollstein, MD, UPMC Eye Center, Eye and Ear Institute, 203 Lothrop St, Pittsburgh, PA 15213, US. E: wollsteing@upmc.edu

Glaucoma is a chronic progressive optic neuropathy, which can lead to irreversible blindness. ${ }^{1}$ It is characterized by loss of retinal nerve tissue that results in visual function deterioration. Structural changes, such as accelerated loss of retinal ganglion cells (RGCS) and their axons, and a specific pattern of damage in the optic nerve head $(\mathrm{ONH})$ associated with visual field (VF) loss, are considered hallmarks of glaucoma. ${ }^{2}$

The efficacy of glaucoma management depends mostly on the ability to reliably detect disease progression as early as possible. Detection of progression allows the clinician to initiate or modify treatment, which can slow glaucoma progression and potentially preserve vision.

Even though optic disc stereophotographs are considered the gold standard for the evaluation of glaucomatous structural damage, the estimation of structural progression in glaucoma using this tool is challenging due to its subjective nature. ${ }^{3}$ The recent evolution of imaging tools noticeably improved the objective and quantitative determination of structural changes and the assessment of progression caused by glaucoma. ${ }^{4-6}$ However, the assessment of structural progression in glaucoma still comprises several dilemmas due to difficulties in differentiating between true glaucomatous change and natural agerelated loss of retinal nerve tissue, measurement variability, and a lack of widely accepted and efficient gold standard criteria for establishing glaucoma progression. In this review we will summarize the imaging tools that are currently available, namely scanning laser polarimetry (SLP), confocal scanning laser ophthalmoscopy (CSLO), and spectral domain optical coherence tomography (SD-OCT). We will focus particularly on their ability to detect glaucoma progression, as well as the strengths and limitations of each imaging technology.

\section{Detection of Glaucoma Progression}

Progression of glaucoma can be estimated by subjective assessment based on clinical experience and judgment, or by statistical analysis of quantitative measurements. The statistical methods used to detect glaucoma progression can be classified as event- or trend-based analysis.

In event-based analysis a series of follow-up measurements are compared with baseline measurements, and progression is defined when measurements exceed a predetermined threshold for change from baseline. This method is highly sensitive to the threshold level, with a higher threshold resulting in fewer cases identified as progressing with only a small number of false positive classifications and vice versa when choosing a lower threshold. The threshold is often selected based on the physical properties of the imaging device (e.g., measurement reproducibility), or by population-based data. The main disadvantage of this method is the effect of erroneous deviating measurements that might be labeled as progression. A confirmatory second test can resolve most of the false progression classifications in this method.

Trend-based analysis uses regression analysis or mixed effect regression analysis to estimate the rate of change in the examined parameters. Progression is defined either when the rate of change is significantly different than a no change slope (zero slope), or when the rate is different than population or individually derived slope. This method 
is less affected by measurement errors at a given time point than the event-based method, but it requires a large number of tests in order to be considered reliable. As this method provides a slope, this can be extrapolated to predict future progression and risk for the development of functionally significant visual deficits, thus impacting glaucoma management. The main disadvantages of trend-based analysis are the requirement for a large number of visits and the risk for ignoring true progression events when changes initially occur. The last disadvantage is due to the neutralization of sudden progression events by the overall rate of change. This can lead to a delay in identifying progression that would lead to the inappropriate management of glaucoma. Moreover, the basic assumption for trend-based analysis is that progression is a linear process, which might not be true for all eyes.

Another approach for the detection of progression is using permutation analysis while assessing the parameters of interest. ${ }^{7}$ In this method, the chronologic order of the measurements are randomly shuffled and compared with the actual rate of change over time. The advantage of this method is that it uses individual subject measurements in order to define the measurement variability rather than relying on population-based information, which might best reflect the actual disease behavior for given patient. However, this method also requires a large number of tests in order to provide reliable limits.

\section{Confocal Scanning Laser Ophthalmoscopy}

CSLO is a noninvasive imaging technology that uses a $670 \mathrm{~nm}$ wavelength diode laser and confocal optics to obtain high-resolution threedimensional (3D) images of the $\mathrm{ONH}$ region. The built-in software merges the stacks of acquired parallel scans into a 3D image, and determines the height of the surface along the optic disc and peripapillary retina. After the operator marks the $\mathrm{ONH}$ margin, the device can compute a variety of stereometric parameters, including disc area, rim area and volume, cup-to-disc ratio, etc. The device can also generate fully automated structural measurements by fitting the pre-defined configuration of the $\mathrm{ONH}$ region to the scan.

The most recent commercially available CSLO device is the Heidelberg Retina Tomograph III (HRT III; Heidelberg Engineering, Heidelberg, Germany). Previous studies have shown rim area to be the most repeatable parameter, both with between-visit and between-operator variability, and it has demonstrated good Iongitudinal reproducibility. ${ }^{8-10}$ Therefore, this parameter is often considered to be the best candidate among the stereometric parameters provided by the device to identify and track glaucomatous changes.

The topographic change analysis (TCA) is currently the primary algorithm for assessing glaucomatous progression available in the HRT III. It is an event-based analysis that identifies topographic surface height change exceeding a predefined threshold level across the entire scanned area of the $\mathrm{ONH}$ and peripapillary region. TCA estimates significant and repeatable changes at the superpixel ( $4 \times 4$ pixels) level using the analysis of variance technique, and displays results in a color-coded map, with red indicating depression and green indicating elevation compared with baseline (see Figure 1). The location of the labeled clusters should be considered when evaluating the TCA report. Clusters within the boundaries of the $\mathrm{ONH}$ should be considered primarily, while clusters along blood vessels should be ignored as they are mostly induced by vascular pulsation. ${ }^{11}$

The concordance between the TCA analysis and optic disc stereophotography in the detection of glaucoma progression varies from $65 \%$ to $82.3 \%$ as reported in different studies. ${ }^{12-14}$ Some studies reported that TCA analysis detects glaucoma progression earlier than VF ${ }^{11}$ and SD-OCT retinal nerve fiber layer (RNFL) thickness examination, ${ }^{15}$ although more than $70 \%$ of TCA progressors did not progress by VF during a 6-year-long follow-up period ${ }^{16}$ while nearly $40 \%$ of TCA stable eyes showed optic disc stereophotographic or VF progression. ${ }^{17}$

For a median follow-up period of 4.0 years, sixty-one stable glaucomatous eyes were prospectively followed. During this period the participants had periodical HRT imaging and VF and optic disc photography. ${ }^{17}$ During the follow-up period, $38.4 \%$ of the eyes that were defined as TCA-stable showed VF and/or photographic progression while only in $22.9 \%$ of eyes that were labeled as TCA-progression there was evidence of VF or photographic progression. This discrepancy might indicate a limited performance of this method or the often observed discordance between progression detected among different analysis methods.

\section{Spectral Domain Optical Coherence Tomography}

SD-OCT obtains real-time, high-resolution, cross-sectional tissue scans at the micrometer scale. This iteration of the technology is based on analyzing the frequency information of back reflected light from the eye generated by a near-infrared broad-bandwidth light source that is detected by a charge-coupled device (CCD) camera and a spectrometer. ${ }^{18}$ The frequency information is converted into intensity profiles using Fourier-transformation, allowing the acquisition of cross-sectional scans of tissue with rapid scanning speed. Fast scanning rate (currently, up to 75,000 axial scans/sec) allows swiping through the area of interest to acquire 3D data. The glaucoma module obtains RNFL thickness data from a $3.46 \mathrm{~mm}$ diameter peripapillary circle centered on the optic disc, peripapillary RNFL thickness map, and $\mathrm{ONH}$ measurements.

There are several commercially available SD-OCT devices, and at the time of writing only some of them offer built-in progression analysis software: the Cirrus HD-OCT (Carl Zeiss Meditec, Dublin, CA), Spectralis OCT (Heidelberg Engineering, Heidelberg, Germany), RTVue Premier (Optovue, Fremont, CA), and Topcon 3D OCT-2000 (Topcon, Tokyo, Japan). All these devices perform trend-based progression analysis, using linear regression, to compute the rate of change of RNFL thickness (global or regional) over time. This rate of change can also be used to predict future progression beyond the most recent visit, and allows the clinician to set goals for treatments.

Currently, only Cirrus HD-OCT and Spectralis OCT provide event-based analysis (the principles of this method were explained above) in addition to the regression analysis. The guided progression analysis (GPA) of Cirrus HD-OCT estimates disease progression by both event- and trendbased analysis. Event analysis is performed on RNFL thickness and change maps (see Figure 2a) and the circumpapillary RNFL thickness profiles (see Figure $2 c$ ). Locations where RNFL thickness was thinner than baseline beyond the level of the population-derived reproducibility 


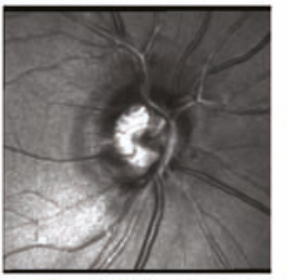

Baseline: Sep/ '2009

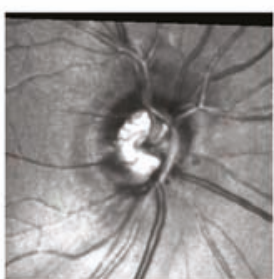

Follow-Up: \#2, Mari 12010

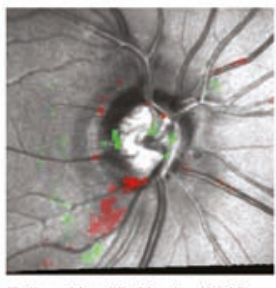

Follow-Up: \#5, Nov' '2010

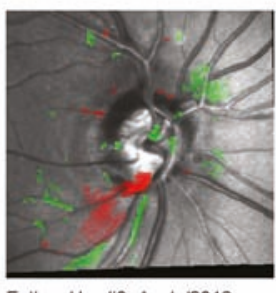

Follow-Up: \#8, Apr/ 12012
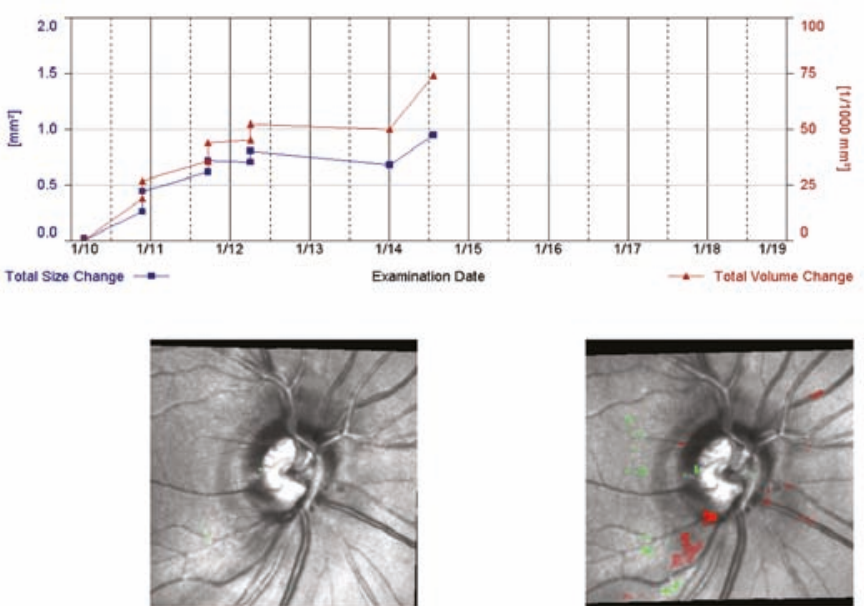

Follow-Up: \#3, Mari '2010

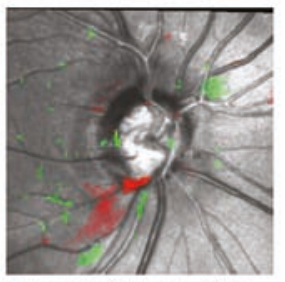

Follow-Up: \#6, Sep/ 12011

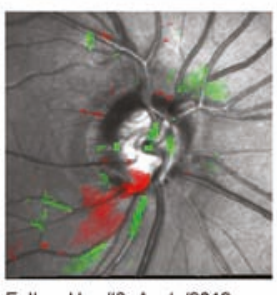

Follow-Up: \#9, Apr/ /2012

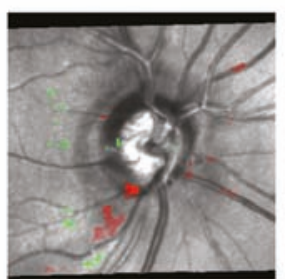

Follow-Up: \#4, Novi 12010

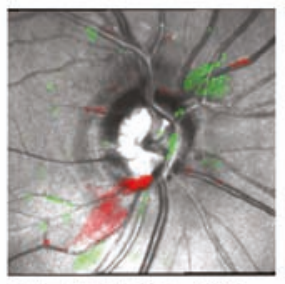

Follow-Up: \#7, Sep/. 1/2011

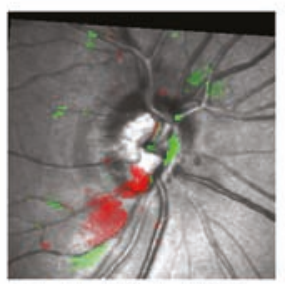

Follow-Up: \#10, Jan/ 12014

A surface height depression (red pixels) is seen in the inferotemporal region that gradually expands during the period of follow-up.

error are highlighted. In the uppermost panel (see Figure 2a) two baseline (left) and two final exams (right) are presented. The full sequence of scans acquired over the period of follow-up is presented in the bottom panel (see Figure 2e). Color-coded results on the RNFL thickness change map and the RNFL thickness profiles mark the first occurrence of statistically significant change in yellow, while consecutive RNFL thinning in the same location is shown in red. It should be noted that in the case presented in Figure 2, a significant change was initially detected in visit \#3, and therefore the area marked in the inferior region (see Figure 2a) is labeled in red in both of the last visits (right). The trend analysis for RNFL thickness change (overall and in hemifields) and the ONH's cup-to-disc ratio over the course of the follow-up period are reported in Figure $2 b$

The RNFL Change Report of the Spectralis OCT provides event-based analysis of circumpapillary RNFL thickness profiles and trend-based analysis of average and sectoral circumpapillary RNFL thickness. The analysis uses the same color code (yellow for the first event and red for the next consecutive event in the same location) to show significant progression from baseline.
SD-OCT has been shown to be more repeatable and reproducible than the earlier generations of the technology, time-domain OCT (TD-OCT), ${ }^{19-23}$ and optic disc stereophotography. ${ }^{24}$ This enables the identification of smaller structural changes with higher confidence and improves the ability to detect changes. ${ }^{25}$

A longitudinal study of 186 glaucomatous eyes reported that the most common pattern of RNFL progression was the widening of RNFL defects, followed by the development of a new RNFL defect, and then a deepening of preexisting defects. ${ }^{26}$ Therefore, the ability of SD-OCT to provide information from the entire peripapillary region further improves the chances of detecting progression. The same study revealed that RNFL progression most commonly occurs in inferotemporal meridians at $2 \mathrm{~mm}$ from the optic disc center, which supports the observation that the superonasal VF frequently deteriorates faster as glaucoma progresses. ${ }^{27}$

SD-OCT technology showed poor agreement with optic disc stereophotography and VF testing in the detection of glaucoma progression, ${ }^{25,28,29}$ similar to TD-OCT, ${ }^{30-32}$ which might be due to spatial 
Figure 2: Spectral Domain Optical Coherence Tomography Guided Progression Analysis

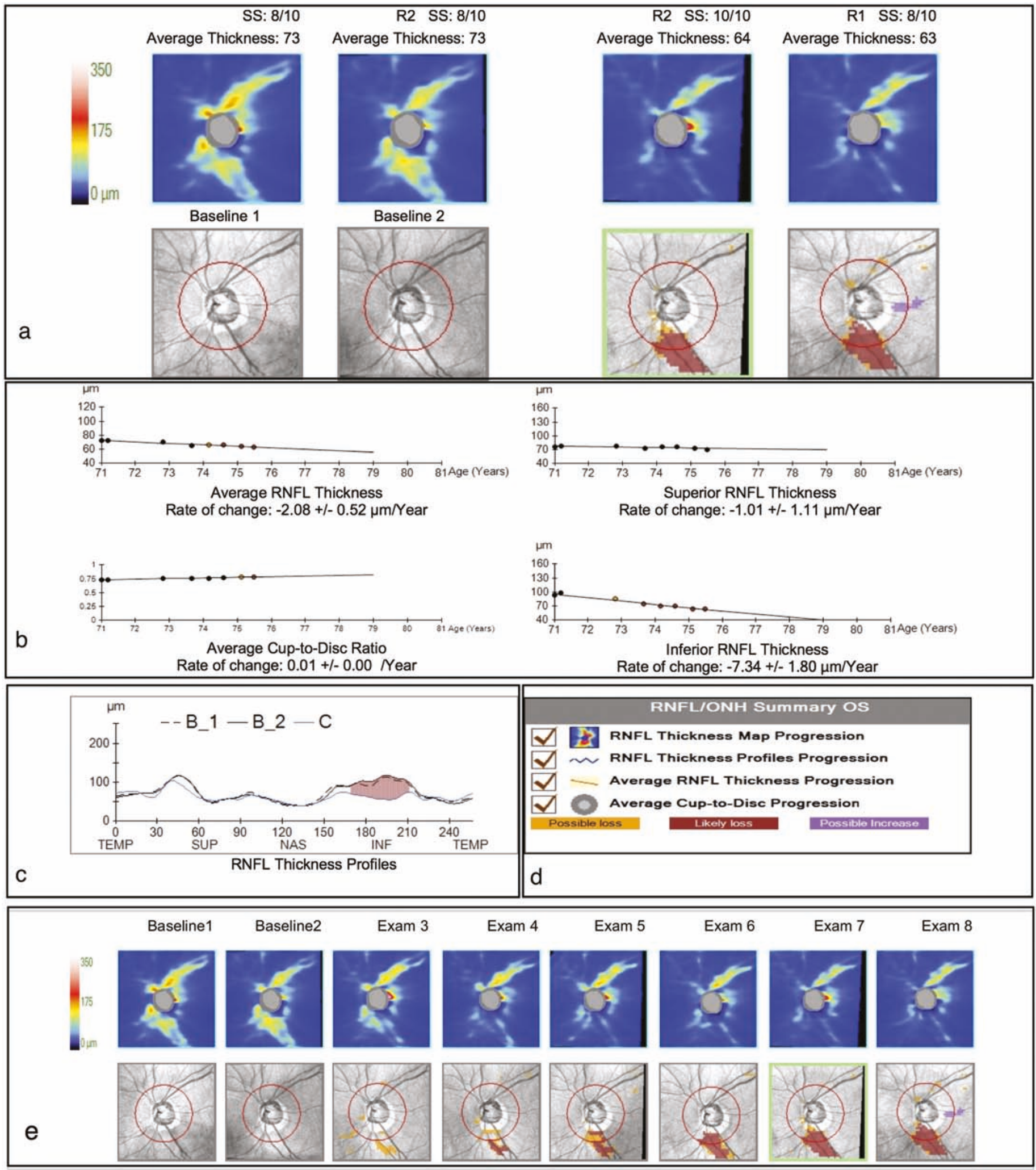

(a) Significant retinal nerve fiber layer (RNFL) thinning marked in the inferior region based on event-based analysis. (b) Significant rate of change is noted in the average and inferior RNFL along with the cup-to-disc ratio, but no change is evident in the superior RNFL hemifield. (c) RNFL thickness profile highlights a corresponding area of thinning in the inferior region. The bottom figure (e) demonstrates the course of RNFL progression in eight consecutive visits. INF = inferior; NAS = nasal; ONH = optic nerve head; SUP = superior: TEMP = temporal. 


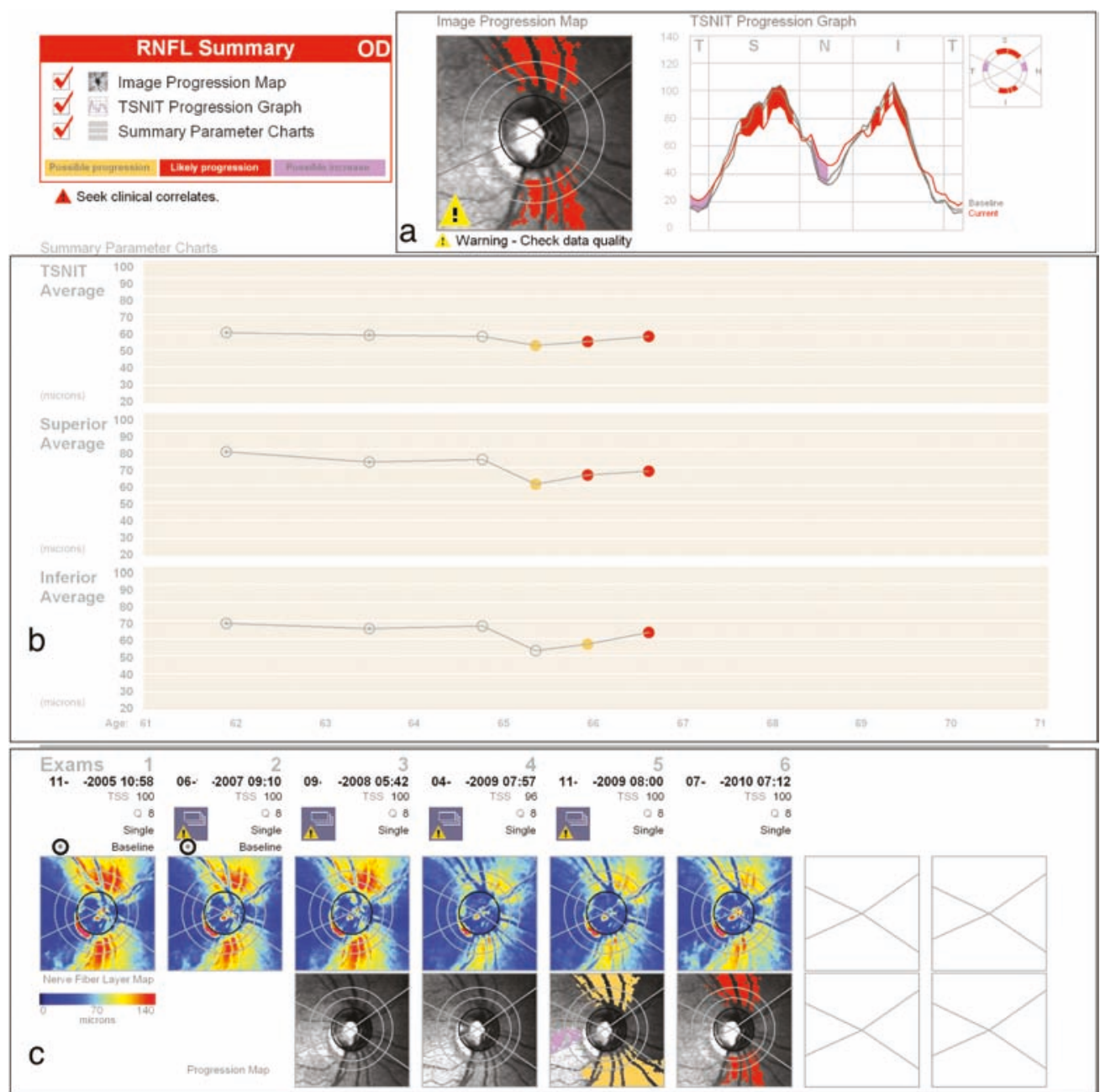

The Image Progression Map (a left, c) and Temporal - Superior - Nasal - Inferior -Temporal (TSNIT) Progression Graph (a right) show progression in the inferior and superior regions. Similarly, the Summary Parameter Charts (b) show a significant rate of thinning of the TSNIT average, superior, and inferior retinal nerve fiber layer (RNFL).

discrepancies between structure and function progression or the timing of progression in different parts or tissues in the eye.

A retrospective study of 272 glaucomatous eyes evaluated the performance of Cirrus HD-OCT GPA for detection of glaucoma progression. ${ }^{33}$ Expert assessment of optic disc photographs, VF GPA, or VF index linear regression analysis were used as reference standards of progression. The authors reported a sensitivity of $25 \%$ and specificity of $88.61 \%$ for the GPA. At the time of this writing there are no published studies on the performance of the automated progression algorithms of other commercially available SD-OCT devices.

\section{Scanning Laser Polarimetry}

SLP measures RNFL thickness by scanning the retina with a polarized laser beam, which splits into two components while passing through highly organized and parallel structures, such as the RNFL. ${ }^{34}$ These components travel at different velocities, which creates a relative phase shift (retardation). The amount of retardation is proportional to the thickness of the birefringent tissue, thus allowing the instrument to measure the RNFL thickness.
The most recent commercially available SLP instrument, GDX PRO (Carl Zeiss Meditec, Dublin, CA), uses GPA software for the detection of glaucoma progression, similar to the one described above for Cirrus SD-OCT (see Figure 3). The GPA provides both event- and trend-based analyses of RNFL thickness change over a follow-up period. The software can provide two types of analysis, depending on whether one or three scans are obtained at each visit. The "fast mode" compares the two most recent images with the two baselines: change is identified as significant if it exceeds the predetermined measurement variability based on population-derived data. By contrast, the "extended mode" uses a mean of three images at each visit, and identifies significant change based on the measurement variability computed with the permutation analysis described above.

GDx's GPA Image Progression Map and the Temporal - Superior Nasal - Inferior -Temporal (TSNIT) Progression Graph (see Figure 3a) represent an RNFL thickness event-based progression analysis using the same color-codes as in Cirrus HD-OCT. In the bottom part of the printout all the exams from the follow-up period are presented (see Figure $3 \mathrm{c}$ ). Linear regression analysis of mean and hemifield 
RNFL thickness are presented in summary Parameters charts (see Figure 3b).

At the time of writing, there are no published data on the agreement of current generation GDX GPA software with ONH stereophotography or VF examination in detection of glaucoma progression. An observational cohort study of 453 glaucoma, glaucoma suspect, and healthy eyes with mean follow-up of 46 months examined the performance of GDX GPA for detecting glaucoma progression. ${ }^{5}$ Progression was defined by VF GPA and expert evaluation of optic disc stereophotographs. Out of 34 eyes that showed progression by VF and/or optic disc stereophotographs the GDX GPA detected 17 eyes (50\% sensitivity). Fourteen eyes showed progression by GDX GPA for a specificity of $96 \%$.

\section{Conclusion}

Although the imaging tools currently available have shown the capacity to detect and quantify glaucomatous progression, the estimation of glaucoma progression remains clinically challenging. This is due to the variable course of the disease and its slow progression, the lack of a commonly accepted gold standard that can be used to accurately indicate glaucomatous progression, and the device measurement variability when used in serial assessments. Additionally, age-related reduction of peripapillar and macular RNFL thickness ${ }^{30,35,36}$ should be considered while assessing glaucoma progression. As the ability to detect disease progression and the full spectrum of disease severity varies between structural and functional methods, it is recommended to clinically assess progression with both methods. ${ }^{30,37}$
1. Foster PJ, Buhrmann R, Quigley HA, Johnson GJ, The definition and classification of glaucoma in prevalence surveys, $B r J$ Ophthalmol, 2002;86:238-42.

2. Quigley HA, Recognizing structural damage to the optic nerve head and nerve fiber layer in glaucoma, Am J Ophthalmol, 1998:125: 563; author reply 564-5.

3. Jampel HD, Friedman D, Quigley $\mathrm{H}$, et al., Agreement among glaucoma specialists in assessing progressive disc changes from photographs in open-angle glaucoma patients, Am J ophthalmol, 2009;147:39-44.e1.

4. Asaoka R, Strouthidis NG, Kappou V, et al., HRT-3 Moorfields reference plane: effect on rim area repeatability and identification of progression, Br J Ophthalmol, 2009;93:1510-3.

5. Alencar LM, Zangwill LM, Weinreb RN, et al., Agreement for detecting glaucoma progression with the gdx guided progression analysis, automated perimetry and optic disc photography, Ophthalmology, 2010;117:462-70.

6. Kotowski J, Wollstein G, Folio LS, et al., Clinical Use of OCT in Assessing Glaucoma Progression, Ophthalmic Surg Lasers Imaging, 2011;42:S6-S14.

7. Patterson AJ, Garway-Heath DF, Strouthidis NG, Crabb DP, A new statistical approach for quantifying change in series of retinal and optic nerve head topography images, Invest Ophthalmol Vis Sci, 2005:46:1659-67.

8. Strouthidis NG, White ET, Owen VM, et al., Improving the repeatability of Heidelberg retina tomograph and Heidelberg retina tomograph II rim area measurements, Br J ophthalmol, 2005;89:1433-7.

9. Jampel $H D$, Vitale $S$, Ding $Y$, et al., Test-retest variability in structural and functional parameters of glaucoma damage in the glaucoma imaging longitudinal study, J Glaucoma, 2006;15:152-7.

10. Leung CK, Lindsey JD, Crowston JG, et al., Longitudinal profile of retinal ganglion cell damage after optic nerve crush with blue-light confocal scanning laser ophthalmoscopy, Invest Ophthalmol Vis Sci, 2009;50:691-701.

11. Bowd C, Balasubramanian $\mathrm{M}$, Weinreb RN, et al., Performance of confocal scanning laser tomograph topographic change analysis (TCA) for assessing glaucomatous progression, Invest analysis (TCA) for assessing glaucom

12. Chauhan BC, McCormick TA, Nicolela MT, LeBlanc RP, Optic disc and visual field changes in a prospective longitudinal study of patients with glaucoma:comparison of scanning laser tomography with conventional perimetry and optic disc photography, Arch ophthalmol, 2001:119:1492-9.
13. Kourkoutas D, Buys YM, Flanagan JG, et al., Comparison of glaucoma progression evaluated with Heidelberg retina tomograph II versus optic nerve head stereophotographs, Can J Ophthalmol, 2007;42:82-8.

14. Vizzeri G, Weinreb RN, Martinez de la Casa JM, et al., Clinicians agreement in establishing glaucomatous progression using the Heidelberg retina tomograph, Ophthalmology, 2009:116:14-24.

15. Xu G, Weinreb RN, Leung CK, Optic nerve head deformation in glaucoma: the temporal relationship between optic nerve head surface depression and retinal nerve fiber layer thinning, Ophthalmology, 2014;121:2362-70.

16. Chauhan BC, Nicolela MT, Artes PH, Incidence and rates of visual field progression after longitudinally measured optic disc change in glaucoma, Ophthalmology, 2009:116:2110-8.

17. Kourkoutas D, Buys YM, Flanagan JG, et al., Clinical significance of optic disc progression by topographic change analysis maps in glaucoma: An 8-year follow-up study, J Ophthalmol, 2014;2014:987389.

18. Fercher AF, Mengedoht $K$, Werner W, Eye-length measurement by interferometry with partially coherent light, opt Lett, 1988:13:186-8

19. Schuman JS, Spectral domain optical coherence tomography for glaucoma (an AOS thesis), Trans Am Ophthalmol SOC, 2008:106:426-58.

20. Menke MN, Knecht P, Sturm V, et al., Reproducibility of nerve fiber layer thickness measurements using 3D Fourier-domain OCT, Invest Ophthalmol Vis Sci, 2008;49:5386-91.

21. Leung $C K$, Cheung $C Y$, Weinreb RN, et al., Retinal nerve fiber layer imaging with spectral-domain optical coherence tomography: a variability and diagnostic performance study, Ophthalmology, 2009:116:1257-63, 1263.e1-2.

22. Garas $\mathrm{A}$, Vargha $\mathrm{P}$, Holló $\mathrm{G}$, Reproducibility of retinal nerve fiber layer and macular thickness measurement with the RTVue-100 optical coherence tomograph, Ophthalmology, 2009;116:1257$63,1263 . e 1-2$

23. Mwanza JC, Chang RT, Budenz DL, et al., Reproducibility of peripapillary retinal nerve fiber layer thickness and optic nerve head parameters measured with cirrus HD-OCT in glaucomatous eyes, Invest Ophthalmol Vis Sci, 2010;51:5724-30.

24. Sharma A Oakley JD, Schiffman JC, et al, Comparison of automated analysis of Cirrus HD-OCTTM spectral domain optical coherence tomography with stereo photos of the optic disc, Ophthalmology, 2011;118:1348-57.

25. Leung CK, Chiu V, Weinreb RN, et al., Evaluation of retinal nerve fiber layer progression in gaucoma: A comparison between spectral-domain and time-domain optical coherence tomography, Ophthalmology, 2011;118:1558-62.

26. Leung CK, Yu M, Weinreb RN, et al., Retinal nerve fiber layer imaging with spectral-domain optical coherence tomography: patterns of retinal nerve fiber layer progression, Ophthalmology 2012:119:1858-66.

27. Pereira ML, Kim CS, Zimmerman MB, et al., Rate and pattern of visual field decline in primary open-angle glaucoma, Ophthalmology, 2002;109:2232-40.

28. Xin $D$, Greenstein $V C$, Ritch R, et al., A comparison of functional and structural measures for identifying progression of glaucoma, Invest Ophthalmol Vis Sci, 2011:52:519-26.

29. Na JH, Sung KR, Baek S, et al., Progression of retinal nerve fiber layer thinning in glaucoma assessed by cirrus optical coherence tomography-guided progression analysis, Curr Eye Res, 2013:38:386-95

30. Wollstein G, Schuman JS, Price LL, et al., Optical coherence tomography longitudinal evaluation of retinal nerve fiber layer thickness in glaucoma, Arch Ophthalmol, 2005;123: 464-70.

31. Artes $\mathrm{PH}$, Chauhan $\mathrm{BC}$, Longitudinal changes in the visual field and optic disc in glaucoma, Prog Retin Eye Res, 2005:24:333-54.

32. Leung $\mathrm{CK}$, Cheung $\mathrm{CY}$, Weinreb RN, et al., Evaluation of retina nerve fiber layer progression in glaucoma: a study on optical coherence tomography guided progression analysis, Invest Ophthalmol Vis SCi, 2010;51:217-22.

33. Na JH, Sung KR, Baek S, et al., Progression of retinal nerve fiber layer thinning in glaucoma assessed by cirrus optical coherence tomography-guided progression analysis, Curr Eye Res, 2013:38:386-95.

34. Huang X-R, Knighton RW, Microtubules contribute to the birefringence of the retinal nerve fiber layer, Invest Ophthalmol Vis Sci, 2005;46:4588-93.

35. Kim E-J, Hong S, Kim CY, et al., Attenuated age-related thinning of peripapillary retinal nerve fiber layer in long eyes, Korean $\lrcorner$ Ophthalmol, 2011;25:248-51.

36. Alasil T, Wang K, Keane PA, et al., Analysis of normal retinal nerve fiber layer thickness by age, sex, and race using spectral domain optical coherence tomography, J Glaucoma 2013;22:532-41.

37. Strouthidis NG, Scott A, Peter NM, Garway-Heath DF, Optic disc and visual field progression in ocular hypertensive subjects: detection rates, specificity, and agreement, Invest Ophthalmo Vis Sci, 2006;47:2904-10. 